PROCEEDINGS OF THE

AMERICAN MATHEMATICAL SOCIETY

Volume 134, Number 5, Pages 1309-1314

S 0002-9939(05)08049-4

Article electronically published on October 4, 2005

\title{
MULTIPLICATION AND DIVISION BY INNER FUNCTIONS IN THE SPACE OF BLOCH FUNCTIONS
}

\author{
DANIEL GIRELA, CRISTÓBAL GONZÁLEZ, AND JOSÉ ÁNGEL PELÁEZ
}

(Communicated by Juha M. Heinonen)

\begin{abstract}
A subspace $X$ of the Hardy space $H^{1}$ is said to have the $f$-property if $h / I \in X$ whenever $h \in X$ and $I$ is an inner function with $h / I \in H^{1}$. We let $\mathcal{B}$ denote the space of Bloch functions and $\mathcal{B}_{0}$ the little Bloch space. Anderson proved in 1979 that the space $\mathcal{B}_{0} \cap H^{\infty}$ does not have the $f$-property. However, the question of whether or not $\mathcal{B} \cap H^{p}(1 \leq p<\infty)$ has the $f$-property was open. We prove that for every $p \in[1, \infty)$ the space $\mathcal{B} \cap H^{p}$ does not have the $f$-property.

We also prove that if $B$ is any infinite Blaschke product with positive zeros and $G$ is a Bloch function with $|G(z)| \rightarrow \infty$, as $z \rightarrow 1$, then the product $B G$ is not a Bloch function.
\end{abstract}

\section{INTRODUCTION AND STATEMENT OF RESULTS}

We denote by $\Delta$ the unit disc $\{z \in \mathbb{C}:|z|<1\}$ and by $H^{p}(0<p \leq \infty)$ the classical Hardy spaces of analytic functions in $\Delta$ (see [6] and [9]). A function $I$, analytic in $\Delta$, is said to be an inner function if $I \in H^{\infty}$ and $I$ has a radial limit $I\left(e^{i \theta}\right)$ of modulus one for almost every $e^{i \theta} \in \partial \Delta$.

Given a function $v \in L^{\infty}(\partial \Delta)$, the associated Toeplitz operator $T_{v}$ is defined by

$$
\left(T_{v} f\right)(z)=\frac{1}{2 \pi i} \int_{\partial \Delta} \frac{v(\zeta) f(\zeta)}{\zeta-z} d \zeta \quad\left(f \in H^{1}, z \in \Delta\right) .
$$

Definition 1.1. A subspace $X$ of $H^{1}$ is said to have the $K$-property if $T_{\bar{\psi}}(X) \subset X$ for any $\psi \in H^{\infty}$.

Definition 1.2. A subspace $X$ of $H^{1}$ is said to have the $f$-property if $h / I \in X$ whenever $h \in X$ and $I$ is an inner function with $h / I \in H^{1}$.

These notions were introduced by Havin 12 and Korenblum [14. The $K$ property implies the $f$-property: indeed, if $h \in H^{1}, I$ is inner and $h / I \in H^{1}$, then $h / I=T_{\bar{I}} h$.

In addition to the Hardy spaces $H^{p}(1<p<\infty)$ many other spaces such as the Dirichlet space $\mathcal{D}([12],[14)$ and several spaces of Dirichlet type (see [7, [15] and

Received by the editors April 22, 2004 and, in revised form, November 17, 2004.

2000 Mathematics Subject Classification. Primary 30D45, 30D50, 30 D55.

Key words and phrases. Bloch functions, inner functions, Blaschke products, the $f$-property, the $K$-property, Toeplitz operators.

This research has been partially supported by a grant from "La Junta de Andalucía" (FQM210) and by an MCyT grant BFM2001-1736, Spain. 
[19]), the spaces $B M O A$ and $V M O A\left[13\right.$ and the spaces $Q_{p}(0<p<1)$ [8], have the $K$-property.

Clearly, $H^{1}$ has the $f$-property but an argument of duality shows that it does not possess the $K$-property. Hedenmalm proved in 13 that $V M O A \cap H^{\infty}$ has the $f$-property but does not have the $K$-property. More generally, it is proved in [13] that no subspace of $H^{\infty}$ containing the disc algebra has the $K$-property.

The first example of a space not possessing the $f$-property was given by $\mathrm{Gu}$ rarii [11 who proved that the space of analytic functions in $\Delta$ with an absolutely convergent power series does not have the $f$-property. if

Recall that if $f$ is an analytic function in $\Delta$, then $f$ is said to be a Bloch function

$$
\|f\|_{\mathcal{B}} \stackrel{\text { def }}{=}|f(0)|+\sup _{z \in \Delta}\left(1-|z|^{2}\right)\left|f^{\prime}(z)\right|<\infty .
$$

The space of all Bloch functions is denoted by $\mathcal{B}$. The little Bloch space $\mathcal{B}_{0}$ consists of those $f \in \mathcal{B}$ such that $\lim _{|z| \rightarrow 1}\left(1-|z|^{2}\right)\left|f^{\prime}(z)\right|=0$. Alternatively, $\mathcal{B}_{0}$ is the closure of the polynomials in the Bloch norm. We mention 2] for the theory of Bloch functions. Let us recall that $H^{\infty} \subsetneq B M O A \subsetneq \mathcal{B}$ and $V M O A \subsetneq \mathcal{B}_{0}$.

Anderson proved in [1] that $\mathcal{B}_{0} \cap H^{\infty}$ does not have the $f$-property. Consequently, the same is true for $\mathcal{B}_{0} \cap H^{p}$ for every $p \in[1, \infty)$. It is natural to ask the following question: Does $\mathcal{B} \cap H^{p}(1 \leq p \leq \infty)$ have the $f$ - or $K$-property?

Since $H^{\infty} \subset \mathcal{B}$, we see that $\mathcal{B} \cap H^{\infty}=H^{\infty}$ which has the $f$-property but does not have the $K$-property. It is easy to prove the following result.

Proposition 1.3. If $1 \leq p<\infty$, then $\mathcal{B} \cap H^{p}$ does not have the $K$-property.

In fact, we can prove the following stronger result.

Theorem 1.4. If $1 \leq p<\infty$, then $\mathcal{B} \cap H^{p}$ does not have the $f$-property.

Next we shall consider products of the form $B \cdot f$ with $B \in H^{\infty}$ and $f \in \mathcal{B}$ but before doing so it is convenient to recall some definitions and results. If a sequence of points $\left\{a_{n}\right\}$ in $\Delta$ satisfies the Blaschke condition $\sum_{n=1}^{\infty}\left(1-\left|a_{n}\right|\right)<\infty$, the corresponding Blaschke product $B$ is defined as

$$
B(z)=\prod_{n=1}^{\infty} \frac{\left|a_{n}\right|}{a_{n}} \frac{a_{n}-z}{1-\overline{a_{n}} z} .
$$

Such a product is analytic in $\Delta$, and, in fact, it is an inner function. If there exists a positive constant $\delta$ such that $\prod_{m \neq n}\left|\frac{a_{n}-a_{m}}{1-\bar{a}_{n} a_{m}}\right| \geq \delta$, for all $n$, we say that the sequence $\left\{a_{n}\right\}$ is uniformly separated and that $B$ is an interpolating Blaschke product. Equivalently,

$$
B \text { is an interpolating Blaschke product } \Leftrightarrow \inf _{n \geq 1}\left(1-\left|a_{n}\right|^{2}\right)\left|B^{\prime}\left(a_{n}\right)\right|>0 .
$$

Thus no interpolating Blascke product belongs to $\mathcal{B}_{0}$. Sarason [20] proved that $\mathcal{B}_{0}$ contains infinite Blaschke products. Other constructions of such products were given by Stephenson in 21 and Bishop in [3, where a description of all $H^{\infty}$ functions in $\mathcal{B}_{0}$ is also given.

We recall that a function $f$ which is meromorphic in $\Delta$ is a normal function in the sense of Lehto and Virtanen if

$$
\sup _{z \in \Delta}\left(1-|z|^{2}\right) \frac{\left|f^{\prime}(z)\right|}{1+|f(z)|^{2}}<\infty .
$$


We refer to [2] and [17] for the theory of normal functions. Certainly, any Bloch function is normal. Using (1.1) we can deduce the following result.

Proposition 1.5. If $B$ is an interpolating Blaschke product whose sequence of zeros is $\left\{a_{n}\right\}_{n=1}^{\infty}$ and $G$ is an analytic function in $\Delta$ with $G\left(a_{n}\right) \rightarrow \infty$, as $n \rightarrow \infty$, then the function $f=B \cdot G$ is not normal (and, hence, it is not a Bloch function).

Proposition 1.5 has been used by several authors (see e.g. [16, [5], 22], 23], [10] and 4]) to construct distinct classes of non-normal functions. We can prove a result of this kind dealing with Blaschke products with zeros in a radius but not necessarily interpolating.

Theorem 1.6. Let $B$ be an infinite Blaschke product whose sequence of zeros $\left\{a_{n}\right\}$ is contained in the radius $(0,1)$ and let $G$ be a Bloch function such that $G(z) \rightarrow \infty$, as $z \rightarrow 1$. Then the function $f=B \cdot G$ is not a Bloch function.

\section{DiVISION BY INNER FUNCTIONS}

Even though Proposition 1.3 follows from Theorem 1.4, we shall give a direct proof of it. We shall use the following easy lemma.

Lemma 2.1. The space $\mathcal{B}_{0} \cap H^{p}(1 \leq p<\infty)$ is the closure of the polynomials in $\mathcal{B} \cap H^{p}$, that is, for any $f \in \mathcal{B}_{0} \cap H^{p}$ there exists a sequence of polynomials $\left\{P_{n}\right\}_{n=1}^{\infty}$ which converges to $f$ both in the Bloch norm and in the $H^{p}$-norm.

Proof. Take $f \in \mathcal{B}_{0} \cap H^{p}$ with $1 \leq p<\infty$. For $0<r<1$, set $f_{r}(z)=f(r z)(z \in \Delta)$. Also set $r_{n}=1-\frac{1}{n}, n=1,2, \ldots$ Using Theorem 2.1 of [2] and Theorem 2.6 of [6], we see that

$$
\max \left(\left\|f-f_{r_{n}}\right\|_{\mathcal{B}},\left\|f-f_{r_{n}}\right\|_{H^{p}}\right) \longrightarrow 0, \quad \text { as } n \rightarrow \infty .
$$

For each $n, f_{r_{n}}$ is analytic in $\{|z| \leq 1\}$, hence, we can find a polynomial $P_{n}(z)$ such that $\left|f_{r_{n}}(z)-P_{n}(z)\right|<\frac{1}{n}$ for $|z| \leq 1$. Note that if $g \in H^{\infty}$, then $\|g\|_{\mathcal{B}} \leq 2\|g\|_{H^{\infty}}$ (see p. 13 of [2]) and $\|g\|_{H^{p}} \leq\|g\|_{H^{\infty}}$. This and (2.1) give

$$
\begin{aligned}
\max \left(\left\|f-P_{n}\right\|_{\mathcal{B}},\right. & \left.\left\|f-P_{n}\right\|_{H^{p}}\right) \\
& \leq \max \left(\left\|f-f_{r_{n}}\right\|_{\mathcal{B}},\left\|f-f_{r_{n}}\right\|_{H^{p}}\right)+\frac{2}{n} \underset{n \rightarrow \infty}{\longrightarrow} 0 .
\end{aligned}
$$

Proof of Proposition 1.3 . Take $p \in[1, \infty)$ and suppose that $\mathcal{B} \cap H^{p}$ has the $K$ property. Then for any inner function $I$, we would have that $T_{\bar{I}}\left(\mathcal{B} \cap H^{p}\right) \subset \mathcal{B} \cap H^{p}$. Using the closed graph theorem, it would follow that $T_{\bar{I}}$ is continuous from $\mathcal{B} \cap H^{p}$ into itself. Now, bearing in mind that $T_{\bar{I}}$ maps polynomials into polynomials and using Lemma 2.1. we would deduce that $T_{\bar{I}}\left(\mathcal{B}_{0} \cap H^{p}\right) \subset \mathcal{B}_{0} \cap H^{p}$ for any inner function $I$. Hence, $\mathcal{B}_{0} \cap H^{p}$ would possess the $f$-property. But, as mentioned above, this is not true.

Before embarking into the proof of Theorem 1.4 let us note that if $f$ is an analytic function in $\Delta$ we shall set

$$
M_{\infty}(r, f)=\sup _{|z| \leq r}|f(z)|, \quad 0<r<1 .
$$


Proof of Theorem 1.4. Let $B$ be an infinite Blaschke product in $\mathcal{B}_{0}$ whose sequence of zeros $\left\{a_{n}\right\}_{n=1}^{\infty}$ contains a subsequence which tends to 1 . Set

$$
\varphi(r)=\sup _{r \leq|z|<1}\left(1-|z|^{2}\right)\left|B^{\prime}(z)\right|, \quad \phi(r)=\frac{1}{\varphi(r)}, \quad 0 \leq r<1 .
$$

Since $B \in \mathcal{B}_{0}$, it follows that $\phi(r) \uparrow \infty$, as $r \uparrow 1$.

Let $\left\{\gamma_{n}\right\}_{n=1}^{\infty}$ be a decreasing sequence of positive numbers with $0<\gamma_{n}<1$ for all $n$ such that if

$$
D=\Delta \cup\left(\bigcup_{n=1}^{\infty}\left\{z \in \mathbb{C}:|\operatorname{Im} z|<\gamma_{n}, \operatorname{Re} z>0, n \leq|z|<n+1\right\}\right)
$$

and $F$ is the conformal mapping from $\Delta$ onto $D$ with $F(0)=0$ and $F^{\prime}(0)>0$, then

$$
M_{\infty}(r, F)=\mathrm{O}(\phi(r)), \quad \text { as } r \rightarrow 1 .
$$

Such a sequence $\left\{\gamma_{n}\right\}$ can be constructed using the Carathèodory kernel theorem (see Theorem 3.2 of [4] and its proof). Since $\operatorname{Im} F$ is bounded, it follows that

$$
F \in B M O A \subset \mathcal{B} \text {. }
$$

Also, it is clear that

$$
F(z) \rightarrow \infty, \quad \text { as } z \rightarrow 1
$$

Set

$$
f(z)=B(z) F(z), \quad z \in \Delta .
$$

Using (2.2), (2.4), (2.5) and bearing in mind that $|B(z)| \leq 1$, we deduce that there exist two positive constants $C_{1}$ and $C_{2}$ such that

$$
\begin{aligned}
\left(1-|z|^{2}\right)\left|f^{\prime}(z)\right| & \leq\left(1-|z|^{2}\right)\left|B^{\prime}(z)\right||F(z)|+\left(1-|z|^{2}\right)\left|F^{\prime}(z)\right||B(z)| \\
& \leq C_{1} \varphi(|z|) \phi(|z|)+C_{2}=C_{1}+C_{2},
\end{aligned}
$$

for all $z \in \Delta$. Hence, $f \in \mathcal{B}$. Note that (2.5) implies that $F \in H^{p}$ for all $p<\infty$, which, since $B$ is bounded, implies that the same is true for $f$. Consequently, we have proved that

$$
f \in \mathcal{B} \cap H^{p}, \quad 1 \leq p<\infty .
$$

Now let $\left\{a_{n_{k}}\right\}_{k=1}^{\infty}$ be a subsequence of the sequence $\left\{a_{n}\right\}$ such that

$$
a_{n_{k}} \rightarrow 1, \quad \text { as } k \rightarrow \infty,
$$

and

$$
\left\{a_{n_{k}}\right\}_{k=1}^{\infty} \text { is uniformly separated. }
$$

Let $B_{1}$ be the Blaschke product whose sequence of zeros is $\left\{a_{n_{k}}\right\}_{k=1}^{\infty}$ and set

$$
B_{2}=\frac{B}{B_{1}}, \quad g=\frac{f}{B_{2}}=B_{1} F .
$$

It is clear that $B_{2}$ is a Blaschke product and that $g \in H^{p}$ for all $p<\infty$. Next we are going to prove that $g \notin \mathcal{B}$. Clearly, this implies that $\mathcal{B} \cap H^{p}(1 \leq p<\infty)$ does not possess the $f$-property.

Since $\left\{a_{n_{k}}\right\}$ is uniformly separated, there exists $A>0$ such that

$$
\left(1-\left|a_{n_{k}}\right|^{2}\right)\left|B_{1}^{\prime}\left(a_{n_{k}}\right)\right| \geq A, \quad \text { for all } k \text {. }
$$


Then, using (2.9), (2.6), (2.11) and Proposition 1.5, we deduce that $g=f / B_{2}$ is not a Bloch function.

\section{Multiplication by Blaschke products with zeros in a Radius}

Proof of Theorem 1.6. Let $\left\{a_{n}\right\}, B$ and $G$ be as in the statement of Theorem 1.6 and set $g=B G$. Take $\alpha \in \Delta \backslash(-1,1)$ such that $\alpha$ is not in the set $\{B(a): a \in$ $\left.\Delta, B^{\prime}(a)=0\right\}$. Clearly, $B((0,1)) \subset(-1,1)$ and, hence, $\alpha$ is not a cluster point of $\left.B\right|_{(0,1)}$. Then, using a result of Marshall and Sarason (see [18]), we deduce that the Frostman shift $B_{\alpha}$ defined by

$$
B_{\alpha}(z)=\frac{B(z)-\alpha}{1-\bar{\alpha} B(z)}, \quad z \in \Delta,
$$

is an interpolating Blaschke product. Thus, if $\left\{b_{n}\right\}_{n=1}^{\infty}$ is the sequence of zeros of $B_{\alpha}$, there exists a positive constant $\delta$ such that

$$
\left(1-\left|b_{n}\right|^{2}\right)\left|B_{\alpha}^{\prime}\left(b_{n}\right)\right| \geq \delta, \quad \text { for all } n \text {. }
$$

Also, using Theorem 6.1 on p. 75 of [9], we easily see that $b_{n} \rightarrow 1$, as $n \rightarrow \infty$. Note that there exists two positive constants $A_{1}$ and $A_{2}$ such that

$$
A_{1}\left|B_{\alpha}^{\prime}(z)\right| \leq\left|B^{\prime}(z)\right| \leq A_{2}\left|B_{\alpha}^{\prime}(z)\right|, \quad z \in \Delta .
$$

This and (3.2) give

$$
\begin{aligned}
\left(1-\left|b_{n}\right|^{2}\right)\left|g^{\prime}\left(b_{n}\right)\right| & \geq\left(1-\left|b_{n}\right|^{2}\right)\left|B^{\prime}\left(b_{n}\right)\right|\left|G\left(b_{n}\right)\right|-\left(1-\left|b_{n}\right|^{2}\right)\left|G^{\prime}\left(b_{n}\right)\right|\left|B\left(b_{n}\right)\right| \\
& \geq A_{1}\left(1-\left|b_{n}\right|^{2}\right)\left|B_{\alpha}^{\prime}\left(b_{n}\right)\right|\left|G\left(b_{n}\right)\right|-\|G\|_{\mathcal{B}} \\
& \geq A_{1} \delta\left|G\left(b_{n}\right)\right|-\|G\|_{\mathcal{B}} \rightarrow \infty, \quad \text { as } n \rightarrow \infty .
\end{aligned}
$$

Consequently, $g$ is not a Bloch function.

\section{REFERENCES}

1. J. M. Anderson, On division by inner factors, Comment. Math. Helv. 54, 2 (1979), 309-317. MR:0535061 (81d:30052)

2. J. M. Anderson, J. Clunie and Ch. Pommerenke, On Bloch functions and normal functions, J. Reine Angew. Math. 270 (1974), 12-37. MR0361090 (50:13536)

3. C. J. Bishop, Bounded functions in the little Bloch space, Pacific J. Math. 142 (1990), 209225. MR1042042(91b:30101)

4. O. Blasco, D. Girela and M. A. Márquez, Mean growth of the derivative of analytic functions, bounded mean oscillation, and normal functions, Indiana Univ. Math. J. 47 (1998), no. 3, 893-912. MR1665796 (99m:30068)

5. D. M. Campbell, Nonnormal sums and products of unbounded normal functions. II, Proc. Amer. Math. Soc. 74 (1979), no. 1, 202-203. MR0521899 (81j:30047)

6. P. L. Duren, Theory of $H^{p}$ Spaces (Academic Press, New York-London 1970. Reprint: Dover, Mineola, New York 2000). MR0268655 (42:3552)

7. K. M. Dyakonov, Factorization of smooth analytic functions via Hilbert-Schmidt operators (in Russian), Algebra i Analiz 8 (1996), no. 4, 1-42; translation in St. Petersburg Math. J. 8 (1997), no. 4, 543-569. MR1418253 (97m:46038)

8. K. M. Dyakonov and D. Girela, On $Q_{p}$ spaces and pseudoanalytic extension, Ann. Acad. Sci. Fenn. Ser. A Math. 25 (2000), 477-486. MR1762431 (2001e:30056)

9. J. B. Garnett, Bounded Analytic Functions (Academic Press, New York-London, 1981). MR0628971 (83g:30037)

10. D. Girela, On a theorem of Privalov and normal funcions, Proc. Amer. Math. Soc. 125 (1997), no. 2, 433-442. MR 1363422 (97d:30046)

11. V. P. Gurarii, The factorization of absolutely convergent Taylor series and Fourier integrals (in Russian). Investigations on linear operators and the theory of functions, III, Zap. Naučn. Sem. Leningrad. Otdel. Mat. Inst. Steklov. (LOMI) 30 (1972), 15-32. MR0340622 (49:5374) 
12. V. P. Havin, On the factorization of analytic functions smooth up to the boundary (in Russian), Zap. Nauch. Sem. LOMI. 22 (1971), 202-205. MR0289783 (44:6970)

13. H. Hedenmalm, On the $f$ - and $K$-properties of certain function spaces, Contemporary Math. 91 (1989), 89-91. MR1002590 (90g:46076)

14. B. I. Korenblum, An extremal property of outer functions (in Russian), Mat. Zamet. 10 (1971), 53-56; translation in Math. Notes 10 (1971), 456-458 (1972). MR0288274 (44:5472)

15. B. I. Korenblum and V. M. Faivyshevskii, A certain class of compression operators that are connected with the divisibility of analytic functions (in Russian), Ukrain. Mat. Z. 24 (1972), 692-695; translation in Ukrainian Math. J. 24 (1973), 559-561. MR0320801 (47:9335)

16. P. Lappan, Non-normal sums and products of unbounded normal functions, Michigan Math. J. 8 (1961), 187-192. MR0131554 (24:A1403)

17. O. Lehto and K. J. Virtanen, Boundary behaviour and normal meromorphic functions, Acta Math. 97 (1957), 47-75. MR0087746 (19:403f)

18. K. Y. Li, Interpolating Blaschke products and the left spectrum of multiplication operators on the Bergman space, Hokkaido Math. J. 21 (1992), no. 2, 295-304. MR1169796 (93h:30053)

19. M. Rabindranathan, Toeplitz operators and division by inner functions, Indiana Univ. Math. J. 22 (1972), no. 6, 523-529. MR0306964(46:6085)

20. D. Sarason, Blaschke products in $\mathcal{B}_{0}$, Linear and Complex Analysis Problem Book, Lecture Notes in Math. 1043 (Springer-Verlag, Berlin, Heidelberg, New York, Tokyo 1984), 337-338. MR 0734178 (85k:46001)

21. K. Stephenson, Construction of an inner function in the little Bloch space, Trans. Amer. Math. Soc. 308 (1988), no. 2, 713-720. MR0951624 (89k:30031)

22. S. Yamashita, A nonnormal function whose derivative has finite area integral of order $0<$ $p<2$, Ann. Acad. Sci. Fenn. Ser. A I Math. 4 (1979), no. 2, 293-298. MR0565879 (81k:30042)

23. S. Yamashita, A nonnormal function whose derivative is of Hardy class $H^{p}, 0<p<1$, Canad. Math. Bull. 23 (1980), no. 4, 499-500. MR0602610(82a:30046)

Departamento de Análisis Matemático, Facultad de Ciencias, Universidad de Málaga, Campus de Teatinos, 29071 Málaga, Spain

E-mail address: girela@uma.es

Departamento de Análisis Matemático, Facultad de Ciencias, Universidad de Málaga, Campus de Teatinos, 29071 Málaga, Spain

E-mail address: cmge@uma.es

Departamento de Análisis Matemático, Facultad de Ciencias, Universidad de Málaga, Campus de Teatinos, 29071 Málaga, Spain

E-mail address: pelaez@anamat.cie.uma.es 